\title{
Avaliação da pressão intraocular e exoftalmometria pré e pós-bloqueio peribulbar em pacientes submetidos à facectomia
}

\author{
Intraocular pressure and exotalmometer evaluation \\ before and after peribulbar anaesthesia in cataract surgery
}

Marina Favarato1, Juliana Almodin², Flavia Almodin³, Tadeu Cvintal ${ }^{4}$

\begin{abstract}
ReSUMO
Objetivos: Comparar os efeitos sobre a PIO após o bloqueio peribulbar com lidocaína e bupivacaína em pacientes submetidos à cirurgia de facectomia com implante de lente intraocular (LIO), bem como avaliar indiretamente e comparar a distribuição dos anestésicos na órbita com a medida da exoftalmometria antes e após a anestesia peribulbar. A bupivacaína e a lidocaína são anestésicos locais que rotineiramente têm sido utilizados para realização de bloqueio peribulbar. Sabe-se que a pressão intraocular (PIO) é um fator importante no pré-operatório imediato da cirurgia de catarata para evitar possíveis complicações como perda vítrea e hérnia de íris. Métodos: Cinquenta pacientes de ambos os sexos, com idade entre 48 e 93 anos, com avaliação pré-anestésica ASA I e II, foram randomizados após a conclusão da pesquisa em 2 grupos: Grupo 1: os que receberam lidocaína e Grupo 2: os que receberam bupivacaína. A medida da PIO foi realizada e analisada em 4 momentos: Antes da anestesia; 1 minuto após o bloqueio; 5 minutos após o bloqueio; 7 minutos após o bloqueio. Também foi avaliado a exoftalmometria antes e imediatamente após o bloqueio com exoftalmômetro de Luedde. Resultados: Houve um aumento na medida da PIO com o bloqueio anestésico nos dois grupos comparados, porém não houve diferença estatisticamente significante no aumento da PIO quando comparada as duas drogas analisadas. Quando comparamos a exoftalmometria, identificamos que não houve diferença estatisticamente significante no aumento da proptose em relação aos dois grupos, isto é, os dois anestésicos quando comparados distribuem-se da mesma forma na cavidade orbitária. Conclusão: Não houve diferença na variação do aumento da PIO e da na variação da exoftalmometria entre os dois anestésicos comparados.
\end{abstract}

Descritores: Bloqueio peribulbar; Pressão intraocular; Exoftalmometria; Bupivacaína; Lidocaína

\footnotetext{
${ }^{1}$ Residente do Segundo Ano do Instituto de Oftalmologia Tadeu Cvintal - São Paulo (SP), Brasil;

${ }^{2}$ Assistente do Departamento de Glaucoma do Instituto de Oftalmologia Tadeu Cvintal - São Paulo (SP), Brasil;

${ }^{3}$ Estagiária do Instituto de Oftalmologia Tadeu Cvintal - São Paulo (SP), Brasil;

${ }^{4}$ Diretor e Chefe do Departamento de Córnea do Instituto de Oftalmologia Tadeu Cvintal - São Paulo (SP), Brasil.
}

Departamento de Glaucoma do Instituto de Oftalmologia Tadeu Cvintal - São Paulo (SP), Brasil.

Os autores declaram inexistir conflitos de interesse.

Recebido para publicação em: 9/9/2010 - Aceito para publicação em 1/2/2011 


\begin{abstract}
Purpose: To compare the effects of IOP after peribulbar anaesthesia with lidocain and bupivacain for cataract surgery with intraocular lens implantation and evaluate exoftalmometer results after the same procedure. Bupivacain and lidocain are local drugs used for peribulbar anaesthesia. It is known that intraocular pressure (IOP) is an important factor for cataract surgery to avoid complications like vitreous loss and iris hernia.Methods: Fifty male and female patients, between 48 and 93 years old, with ASA I and II evaluation before anaesthesia were recruted to participate for this study. We had 2 groups for the study. Group 1: lidocain patients and group 2: bupivacain patients. Intraocular pressure was measured in 4 different periods: before peribulbar anaesthesia, 1 minute, 5 minutes and 7 minutes after. We also performed exoftalmometer evaluation before and right after peribulbar anaesthesia with Luedde exoftalmometer. Results: There was higher IOP after peribulbar anaesthesia, but not significant when compared between both drugs. The exoftalmometer evaluation showed that both drugs are equal distributed in orbit and they both cause proptosis of the ocular globe. Conclusion: There was no difference comparing higher intraocular pressure or exoftalmometer evaluation between both drugs.
\end{abstract}

Keywords: Peribulbar anaesthesia; Intraocular pressure; Exoftalmometer; Bupivacain; Lidocain

\section{INTRODUÇÃO}

A cirurgia de catarata tem sido realizada sob diversas técnicas anestésicas, desde anestesia geral, bloqueios regionais como o retrobulbar ou mais recentemente o peribulbar, e até sob anestesia tópica $^{(1)}$.

A grande popularidade do bloqueio peribulbar em anestesia oftalmológica se deve à boa qualidade da anestesia, à baixa morbidade e à alta taxa de sucesso que a técnica oferece ${ }^{(2)}$.

Os anestésicos locais promovem bloqueio motor em diferentes graus de acordo com as concentrações e dose total utilizadas. Em cirurgias realizadas sobre o globo ocular, além de bloqueio sensitivo, é também necessário para realização do ato cirúrgico que haja acinesia proporcionada por paralisia dos músculos extrínsecos do olho ${ }^{(3)}$.

Rotineiramente têm sido utilizados para realização de bloqueio peribulbar os anestésicos locais bupivacaína e lidocaína, que são classificados como anestésicos locais do tipo amida. A bupivacaína tem longa duração, possui efeito anestésico e analgésico, o início de sua ação anestésica varia entre 2 a 10 minutos e seu efeito pode durar até 7 horas em alguns pacientes, o que é aproximadamente $3 \mathrm{x}$ mais tempo que a lidocaína. A bupivacaína racêmica tem se mostrado eficaz para o bloqueio peribulbar ${ }^{(4)}$ e sua principal desvantagem está relacionada à cardiotoxicidade ${ }^{(5,6)}$.

A lidocaína é um fármaco de curta ação, com início de ação em 1 a 5 minutos após infiltração e em 5 a 15 minutos para outras vias de administração. A duração da anestesia é de 1 a 2 horas, dependendo do tipo de bloqueio e da concentração da lidocaína ${ }^{(7)}$.

Sabe-se que a PIO é um fator importante no préoperatório imediato da cirurgia de catarata. Atkinson et al. expressaram a importância de um "olho macio" para evitar as complicações, particularmente a perda vítrea e a hérnia de íris durante a cirurgia de facoemulsificação ${ }^{(8,9)}$.

Este estudo tem como objetivo comparar os efeitos sobre a PIO após o bloqueio peribulbar com lidocaína e bupivacaína em pacientes que serão submetidos à cirurgia de facectomia com implante de LIO, bem como avaliar indiretamente e comparar a distribuição dos anestésicos na órbita com a medida da exoftalmometria antes e após a anestesia peribulbar.

\section{Métodos}

Após aprovação pela Comissão de Ética do Instituto de Oftalmologia Tadeu Cvintal,foram selecionados 50 pacientes de ambos os sexos, com idade entre 48 e 93 anos, com avaliação pré-anestésica ASA I e II, encaminhados à cirurgia de catarata eletiva com implante de LIO, os quais assinaram o termo de consentimento informado. Os pacientes portadores de glaucoma ou que tinham contraindicação ao bloqueio peribulbar foram excluídos.

Todos os pacientes foram submetidos à midríase medicamentosa com colírio mydriacyl ${ }^{\circledR}$ e fenilefrina ${ }^{\circledR}$. Os pacientes não receberam medicação sedativa préanestésica, e foram randomizados após a conclusão da pesquisa, em 2 grupos:

- Grupo 1: os que receberam lidocaína;

- Grupo 2: os que receberam bupivacaína.

Antes da anestesia os pacientes foram submetidos a jejum total de $8 \mathrm{~h}$ a $12 \mathrm{~h}$. Os bloqueios foram todos realizados pelo mesmo médico em estudo cego, para minimizar diferenças nos resultados.

Todos os pacientes receberam previamente 1 gota em cada olho de colírio anestésico (proximetacaína $0,5 \%$ ). Após antissepsia foi realizado bloqueio peribulbar com agulha de $25 \times 6$ gauge, única punção na 
<smiles>CCN(CC)CC(=O)Nc1c(C)cccc1C</smiles>

Figura 1: Estrutura química dos anestésicos

\section{Gráfico 1}

PIO antes da anestesia e após o bloqueio segundo o grupo de estudo

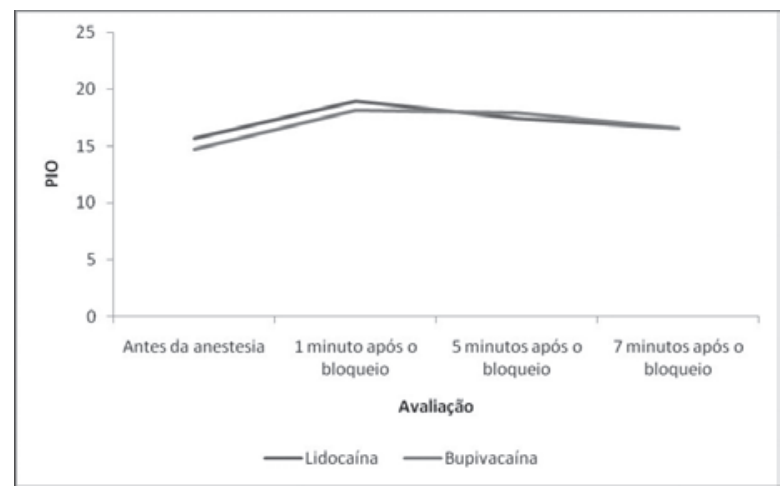

junção do terço lateral com os dois terços mediais da rima orbital inferior, sobre decúbito dorsal, utilizando um volume fixo de $6 \mathrm{ml}$.

No centro cirúrgico, os pacientes foram monitorados continuamente com eletrocardioscópio, oximetria de pulso e pressão arterial não invasiva e submetidos à punção venosa em membro superior com cateter 22 gauge.

A medida da PIO foi realizada com tonômetro de aplanação de Perkins, pelo mesmo médico que realizou o bloqueio e que desconhecia a solução anestésica utilizada. O tonômetro de Perkins é simples, portátil, nãoinvasivo e permite realizar a tonometria de aplanação em decúbito dorsal, facilitando o exame na sala de cirurgia. As medidas foram analisadas em 4 momentos:

- Antes da anestesia;

- 1 mim após o bloqueio;

- 5 minutos após o bloqueio;

- 7 minutos após o bloqueio.

Também foi avaliado a exoftalmometria antes e imediatamente após o bloqueio com exoftalmômetro de Luedde.

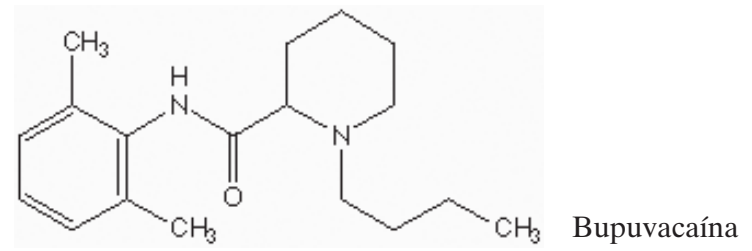

Gráfico 2

Exoftalmometria antes e imediatamente após o
bloqueio segundo o grupo de estudo

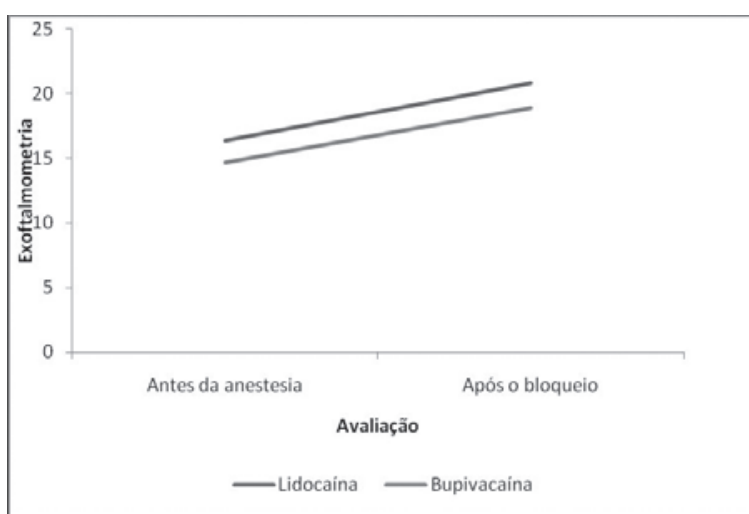

\section{Resultados}

A Tabela 1 relaciona os dados demográficos dos dois grupos em relação ao sexo, idade, pressão arterial sistólica (PAS) e diastólica (PAD), medida da LIO implantada, classificação pré-anestésica ASA e raça.

Os grupos foram comparados quanto à distribuição das variáveis categóricas pelo Teste do qui-quadrado de Pearson ou da razão de verossimilhança quando necessário.

A presença de distribuição normal nas variáveis quantitativas dentro de cada grupo de estudo foi avaliada pelo teste de Kolmogorov-Smirnov.

Foi adotado o nível de significância de $0,05(\mathrm{a}=$ $5 \%$ ) e todas as análises foram realizadas pelo programa estatístico SPSS versão 13.0 for Windows.

Na comparação entre os grupos de anestésico quanto às variáveis demográficas e basais foram encontrados os seguintes resultados:

- homogeneidade quanto à distribuição por sexo $(\mathrm{p}=1,000)$;

- homogeneidade quantoà média de idade $(\mathrm{p}=0,333)$; 
Tabela 1

Caracterização da amostra

\begin{tabular}{|c|c|c|c|}
\hline \multirow[b]{2}{*}{ Variáveis } & \multicolumn{2}{|c|}{ Anestésico } & \multirow[b]{2}{*}{ Valor de $p$} \\
\hline & $\begin{array}{l}\text { Lidocaína } \\
(\mathrm{n}=25)\end{array}$ & $\begin{array}{l}\text { Bupivacaína } \\
\quad(\mathrm{n}=\mathbf{2 5})\end{array}$ & \\
\hline \multicolumn{4}{|l|}{ Sexo $-\mathrm{n}(\%)$} \\
\hline Feminino & $13(52,0)$ & $13(52,0)$ & 1,000 \\
\hline Masculino & $12(48,0)$ & $12(48,0)$ & \\
\hline \multicolumn{4}{|l|}{ Idade (anos) } \\
\hline Média (dp) & $73,1(13,1)$ & $70,1(8,3)$ & 0,333 \\
\hline Mediana & 78 & 70 & \\
\hline Mínimo / Máximo & $48-93$ & $50-86$ & \\
\hline \multicolumn{4}{|l|}{ PAS (mmHg) } \\
\hline Média $(\mathrm{dp})$ & $138,4(13,1)$ & $130,3(15,4)$ & 0,051 \\
\hline Mediana & 137 & 131 & \\
\hline Mínimo / Máximo & $109-160$ & $95-154$ & \\
\hline \multicolumn{4}{|l|}{ PAD (mmHg) } \\
\hline Média $(\mathrm{dp})$ & $76,2(12,3)$ & $70,6(8,0)$ & 0,062 \\
\hline Mediana & 75 & 72 & \\
\hline Mínimo / Máximo & $58-107$ & $54-84$ & \\
\hline Média (dp) & $20,2(4.9)$ & $23,4(3,3)$ & $0,011 *$ \\
\hline Mediana & 20,5 & 23,0 & \\
\hline Mínimo / Máximo & $7-26$ & $18-30$ & \\
\hline \multicolumn{4}{|l|}{ ASA - n (\%) } \\
\hline I & $23(92,0)$ & $24(96,0)$ & 0,548 \\
\hline II & $2(8,0)$ & $1(4,0)$ & \\
\hline \multicolumn{4}{|l|}{ Raça - n (\%) } \\
\hline Branca & $17(68,0)$ & $15(60,0)$ & 0,360 \\
\hline Negra & $1(4,0)$ & $0(0,0)$ & \\
\hline Parda & $7(28,0)$ & $10(40,0)$ & \\
\hline
\end{tabular}

Tabela 2

PIO antes da anestesia e após o bloqueio segundo o grupo de estudo

\begin{tabular}{|c|c|c|c|c|}
\hline \multirow[t]{2}{*}{ Grupo } & \multicolumn{4}{|c|}{ Avaliação } \\
\hline & $\begin{array}{l}\text { Antes da } \\
\text { anestesia }\end{array}$ & $\begin{array}{l}1 \text { minuto após } \\
\text { o bloqueio }\end{array}$ & $\begin{array}{c}5 \text { minutos após } \\
\text { o bloqueio }\end{array}$ & $\begin{array}{l}7 \text { minutos após } \\
\text { o bloqueio }\end{array}$ \\
\hline \multicolumn{5}{|l|}{ Lidocaína (n = 25) } \\
\hline Média $(\mathrm{dp})$ & $15,7(2,5)$ & $18,9(3,7)$ & $17,4(3,5)$ & $16,6(3,1)$ \\
\hline Mínimo - Máximo & $11-20$ & $10-26$ & $11-25$ & $11-24$ \\
\hline \multicolumn{5}{|l|}{ Bupivacaína $(\mathrm{n}=25)$} \\
\hline Média $(\mathrm{dp})$ & $14,8(3,0)$ & $18,1(5,3)$ & $17,9(6,0)$ & $16,6(5,2)$ \\
\hline Mínimo - Máximo & $10-21$ & $12-38$ & $12-43$ & $8-35$ \\
\hline Efeito de grupo & \multirow{2}{*}{\multicolumn{4}{|c|}{$\begin{array}{c}\mathrm{p}=0,778 \\
\mathrm{p}<0.001 *\end{array}$}} \\
\hline Efeito de avaliação & & & & \\
\hline \multirow[t]{2}{*}{ Comparações múltiplas } & $\begin{array}{c}\underset{p}{\operatorname{antes} \times 1 \mathrm{~min}} \\
\mathrm{p}<0,001 *\end{array}$ & \multicolumn{2}{|c|}{$\begin{array}{l}\text { antes } \times 5 \mathrm{~min} \\
\mathrm{p}=0,001 *\end{array}$} & $\begin{array}{c}\text { antes } \times 7 \mathrm{~min} \\
p=0,080\end{array}$ \\
\hline & $1 \mathrm{~min} x 5 \mathrm{~min}$ & \multicolumn{2}{|c|}{$1 \min x 7 \min$} & $5 \min \times 7 \mathrm{~min}$ \\
\hline
\end{tabular}


Tabela 3

\begin{tabular}{|c|c|c|}
\hline \multirow[t]{2}{*}{ Ivaliação } & \multicolumn{2}{|c|}{ Anestésico } \\
\hline & $\begin{array}{l}\text { Lidocaína } \\
\text { (n = 25) }\end{array}$ & $\begin{array}{c}\text { Bupivacaína } \\
(\mathrm{n}=25)\end{array}$ \\
\hline \multicolumn{3}{|l|}{ Antes da anestesia } \\
\hline Média (dp) & $16,4(3,4)$ & $14,7(1,9)$ \\
\hline Mínimo - Máximo & $10-26$ & $11-19$ \\
\hline \multicolumn{3}{|l|}{ Após o bloqueio } \\
\hline Média (dp) & $20,8(3,9)$ & $18,9(2,6)$ \\
\hline Mínimo - Máximo & $12-29$ & $13-23$ \\
\hline
\end{tabular}

- diferença marginalmente significante quanto à média de pressão arterial sistólica $(\mathrm{p}=0,051)$;

- diferença marginalmente significante quanto à média de pressão arterial diastólica $(\mathrm{p}=0,062)$;

- diferença estatisticamente significante quanto à média de LIO $(\mathrm{p}=0,011)$, onde o grupo lidocaína apresentou média de LIO significantemente maior do que a média do grupo bupivacaína;

- homogeneidade quanto à distribuição por ASA $(\mathrm{p}=0,548)$;

- homogeneidade quanto à distribuição por raça $(\mathrm{p}=0,360)$.

Os resultados referentes à medida da $\mathrm{PIO}$ em diferentes momentos são apresentados na Tabela 2.

Foi aplicada a técnica de Análise de Variância com medidas repetidas na análise da PIO com o fator fixo Grupo. As diferenças entre as Avaliações foram localizadas por testes de comparações múltiplas com a correção de Bonferroni.

Não foi encontrada diferença estatisticamente significante entre os grupos quanto às médias de PIO antes da anestesia $(\mathrm{p}=0,247)$.

$\mathrm{Na}$ análise da PIO ao longo do tempo foram observados os seguintes resultados:

- efeito não significante da interação entre os fatores grupo e avaliação $(p=0,228)$, indicando comportamento semelhante ao dos grupos ao longo do tempo;

- efeito não significante do fator grupo $(p=0,778)$;

- efeito estatisticamente significante do fator avaliação $(\mathrm{p}<0,001)$, onde as médias nas avaliações antes e 7 minutos foram semelhantes e significantemente menores do que as médias nas avaliações de 1 minuto e 5 minutos.

O Gráfico 1 mostra a evolução das medidas da PIO ao longo do tempo nos 2 grupos estudados:
Na análise da exoftlamometria foi aplicada a técnica de análise de covariância (ANCOVA) com a medida basal como covariável e o fator fixo Grupo.

Utilizou-se o teste de Mann-Whitney para amostra independentes na análise da variação da exoftalmometria após o bloqueio comparado ao basal.

Foi encontrada diferença estatisticamente significante entre os grupos quanto às médias de exoftalmometria antes da anestesia $(p=0,035)$, indicando que os grupos não são homogêneos na avaliação basal.

Na comparação entre os grupos quanto às médias de exoftalmometria após o bloqueio retirando o efeito das medidas antes da anestesia, não foi encontrada diferença estatisticamente significante entre eles $(p=0,513)$.

Na comparação entre os grupos quanto às médias de D exoftalmometria não foi encontrada diferença estatisticamente significante entre eles $(p=0,629)$.

\section{DısCUSSÃO}

O agente anestésico ideal na cirurgia oftálmica deve oferecer um alto índice terapêutico, com rápido início de ação e duração adequada para cirurgia ${ }^{(10)}$.

Tanto a anestesia retrobulbar quanto a peribulbar podem produzir aumento transitório da PIO e da proptose em função do aumento da pressão intraorbitária pela injeção do anestésico local; o valor da PIO retorna ao nível de controle à medida que ocorre o relaxamento da musculatura extraocular, com decréscimo na pressão extrínseca sobre o globo ${ }^{(11,12)}$.

Nossos resultados mostraram aumento significativo da PIO após o bloqueio peribulbar com bupivacaína e lidocaína, já Serzedo et al. ${ }^{(13)} \mathrm{em}$ seu estudo relataram redução significativa da PIO com uso do anestésico local rupivacaína. Isto pode ser explicado pelo seu efeito vasoconstrictor sobre os vasos da coróide.

Elevações da PIO associadas à anestesia durante a cirurgia intraocular são importantes, porque aumentam a possibilidade de perda do humor vítreo, prolapso do cristalino e hemorragia expulsiva quando o olho é aberto $^{(11)}$.

Neste estudo não foi detectada diferença estatisticamente significante entre os grupos quanto aos valores de PIO antes e após o bloqueio peribulbar, porém houve relevância estatística nos valores ao longo do tempo.

É estatisticamente significante que existe variação ao longo do tempo, isto é, a medida inicial e aos 7 minutos são menores que as medidas no $1^{\circ}$ minuto e $5^{\circ}$ minuto. 
Quando comparamos a exoftalmometria, identificamos que não houve diferença estatisticamente significante no aumento da proptose em relação aos 2 grupos, isto é, os dois anestésicos quando comparados distribuem-se da mesma forma na cavidade orbitária.

\section{Conclusão}

Baseado nos registros tonométricos e exoftalmométricos antes e após o bloqueio anestésico peribulbar, pudemos observar que houve um aumento na medida da PIO e da exoftalmia com o bloqueio anestésico nos dois grupos comparados, fato que se deve ao aumento do volume orbitário, sem aumentar sua área, levando ao aumento da pressão orbitária e da proptose ocular.

Embora seja necessário um estudo mais amplo, com um número maior de casos, este estudo mostra que existe variação da PIO ao longo do tempo.

\section{RefERÊNCIAS}

1. Katayama M, Zambotti HC, Vieira JL, Arrigucci RJ. Bloqueios peribulbar e retrobulbar em cirurgia oftálmica. Estudo clínico comparativo com bupivacaína a 0,755 , com e sem hialuronidase. Rev Bras Anestesiol. 1993;43(3):159-66.

2. Davis DB 2nd, Mandel MR. Posterior peribulbar anaesthesia: an alternative to retrobulbar anaesthesia. Indian J Ophthalmol. 1989;37(2):59-61.

3. Magalhães E, Govêia CS, Oliveira KO. Bupivacaína racêmica, levobupivacaína e ropivacaína em anestesia loco-regional para oftalmologia: um estudo comparativo. Rev Assoc Med Bras (1992). 2004;50(2):195-8.

4. Birt DJ, Cummings GC. The efficacy and safety of $0.75 \%$ levobupivacaine vs $0.75 \%$ bupivacaine for peribulbar anaesthesia. Eye (Lond). 2003;17(2):200-6.
5. Valenzuela C, Snyders DJ, Bennett PB, Tamargo J, Hondeghem LM. Stereoselective block of cardiac sodium channels by bupivacaine in guinea pig ventricular myocytes. Circulation. 1995;92(10):3014-24.

6. Simonetti MPB, Nigro D. Cardiotoxicidade da bupivacaína comparada a lidocaína: tempo de reversão da parada atrial. Rev Bras Anestesiol. 1985;35(1):13-7.

7. Cruz ML, Luna SP, Clark RM, Massone F, Castro GB. Epidural anaesthesia using lignocaine, bupivacaine or a mixture of lignocaine and bupivacaine in dogs. Vet Anaest Analg. 1997;24(1):30-2.

8. Hamilton RC, Gimbel HV, Javitt JC: A prevenção das complicações da anestesia regional em oftalmologia. Clin Oftalmol Am Norte. 1990;3:111-2.

9. Smith GB. Anestesia oftalmológica. Rio de Janeiro: Ed. Colina; 1983. p. 23-6.

10. Soares LF, Helayel PE, Conceição DB, Oliveira Filho GR. Bloqueio peribulbar com a associação da mistura enantiomérica de bupivacaína (S75-R25) a 0,5 e lidocaína a 2: efeitos da adição de hialuronidase. Rev Bras Anestesiol. 2002;52(4):420-5.

11. Johnson RW, Forrest FC. Anaesthesia for ophthalmic surgery. In: Prys-Roberts C, Brown JR BR. International practice of anaesthesia. Oxford: Butterworth Heinemann; 1992. p.1-29.

12. Gjötterberg M, Ingemansson SO. Effect on intraocular pressure of retrobulbar injection of xylocaine with and without adrenaline. Acta Ophthalmol (Copenh). 1977;55(5):709-16.

13. Serzedo PSM, Nociti JR, Zuccolotto EB, Cagnolati CA, Ferreira SB. Pressão intraocular durante bloqueio peribulbar com ropivacaína a 1 por cento. Rev Bras Anestesiol. 2000;50(3):251-3.

\section{Endereço para correspondência \\ Juliana Almodin}

Rua Xavier Curado, $n^{\circ} 351$ apto. 131- Ipiranga

CEP 04210-100 - São Paulo (SP), Brazil

e-mail: Juliana_almodin@hotmail.com 